\title{
SCHWANNOMA RETROPERITONEAL BENIGNO EN PACIENTE CON ANTECEDENTES DE TUMOR DE WILMS EN LA INFANCIA
}

\author{
B. POZO MENGUAL, I. PÉREZ BUSTAMANTE, E. FERNÁNDEZ FERNÁNDEZ, \\ R. MIALDEA FERNÁNDEZ, G. BRIONES MARDONES, M. GARCÍA-COSIO PIQUERAS*, \\ A. ESCUDERO BARRILERO
}

Servicio Urología. *Servicio de Anatomía Patológica. Hospital Ramón y Cajal. Madrid.

Actas Urol Esp. 27 (5): 379-382, 2003

\section{RESUMEN}

"SCHWANNOMA RETROPERITONEAL BENIGNO EN PACIENTE CON ANTECEDENTES DE TUMOR DE WILMS EN LA INFANCIA"

Se presenta un caso de schwannoma benigno retroperitoneal como hallazgo incidental en una joven de 23 años tratada de un tumor de Wilms a los tres años. Se incluye una revisión de la literatura de este tumor tan infrecuente.

PALABRAS CLAVE: Schwannoma. Neoplasias retroperitoneales. Neurilemoma.

\section{ABSTRACT}

"BENIGN RETROPERITONEAL SCHWANNOMA IN PATIENT WITH ANTECEDENTS OF WILM'S TUMOR IN THE INFANCY"

We report a case of benign retroperitoneal schwannoma diagnosed casual in a 23 years old woman treated of a Wilm's tumour twenty years ago. A review of the on this unusual tumour is included. KEY WORDS: Schwannoma. Retroperitoneal neoplasms. Neurilemoma.

$\mathrm{E}$ s conocido que existe un riesgo aumentado de segundas neoplasias en niños tratados por tumor de Wilms. Por un lado la radiación como base de tratamiento hace años y los agentes alquilantes son los que están implicados en la inducción de segundos tumores.

Los investigadores de la National Wilms Tumor Study Group (NWTGS) estiman que existe un riesgo de 8 a 10 veces mayor de segundas neoplasias, con una incidencia acumulada de $1,6 \%$ en 15 años después del tratamiento ${ }^{1}$.

Presentamos un caso de schwannoma retroperitoneal benigno como hallazgo incidental en una joven de 23 años tratada de un tumor de Wilms a los tres años.

\section{CASO CLÍNICO}

Mujer de 23 años de edad, tratada en 1981 por un tumor de Wilms del riñón derecho de histología favorable tipo blastematoso, estadio II (clasificación de la NWTGS). La paciente recibió quimioterapia neoadyuvante con tres ciclos de actinomicina $\mathrm{D}+$ vincristina, posterior nefrectomía radical derecha con linfadenectomía hiliar y trombectomía de vena cava infrahepática. Como tratamiento adyuvante se le dio radioterapia abdominal (dosis total 2000 rads) y quimioterapia (vincristina y ciclofosfamida durante 18 semanas).

La paciente encontrándose asintomática, en una ecografía de control (agosto 2001), se detectó a nivel de hilio renal una masa redondeada, 
homogénea, de aspecto sólido de 4 cms de diámetro, que no se movilizaba con el riñón, situada por delante de la cava, sin afectar a la vía excretora renal; lesión sugestiva de adenopatía retroperitoneal.

La exploración física de la paciente era rigurosamente normal y no presentaba alteraciones analiticas.

Ante los hallazgos se solicitó tomografía axial computarizada + punción aspirativa con aguja fina (TAC+PAAF) de la lesión. En el estudio citológico se apreciaban abundantes células fusiformes con núcleos de variable forma y tamaño y pequeños grupos de células de aspecto epitelial compatible todo ello con una recidiva de su tumor primario, aunque sin certeza diagnóstica por lo que se precisaba estudio histológico e inmunohistoquímico de la pieza quirúrgica.

Informe de la TAC: riñón izquierdo con ectasia precalicial, con adenopatía paraórtica en continuidad con la parte anterior del psoas. No evidencia de metástasis pulmonares, no adenopatías mediastínicas. Parénquima hepático sin metástasis. Nefrectomía derecha (Fig. 1).

Intervención: se realizó laparotomía media suprainfraumbilical, accediendo al retroperitoneo a través del espacio parietocólico izquierdo, se apreciaba una tumoración redondeada de paredes lisas en íntima relación con $4^{\mathrm{a}}$ vértebra lumbar y músculo psoas, lateral a cava y limitada cranealmente por vena renal izquierda. Sobre la tumoración discurría el uréter y la vena gonadal izquierdos. Se extirpó sin dificultad, sin lesionar estructuras adyacentes.

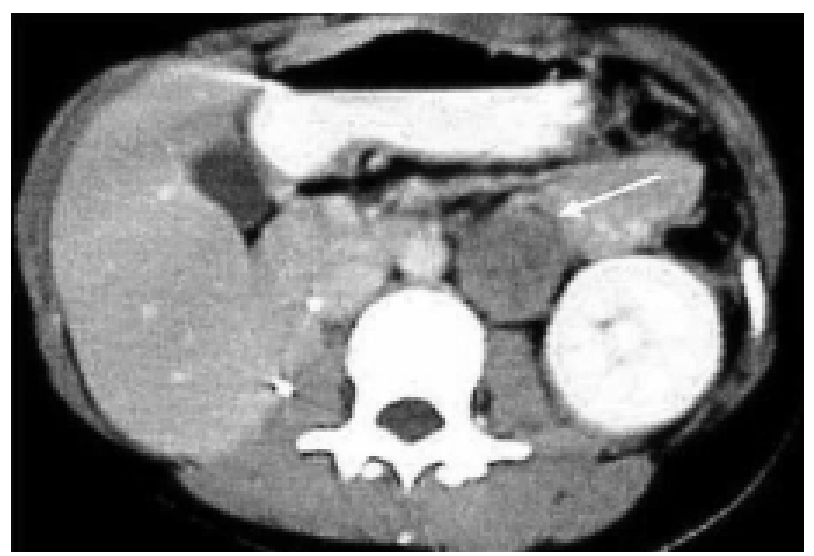

FIGURA 1. TAC abdominopelvico donde se aprecia tumora ción retroperitoneal adyacente a riñón único izquierdo.
La paciente evolucionó satisfactoriamente, permaneciendo asintomática y libre de recidiva a los 6 meses de la cirugía.

\section{HISTOLOGÍA}

Macro-tumoración bien delimitada y aparentemente encapsulada de $6 \mathrm{cms}$ de diámetro máximo, adherida en uno de sus polos a tejido graso. Al corte tiene coloración pardo amarillenta, de distribución irregular con zonas hemorrágicas (Fig. 2).

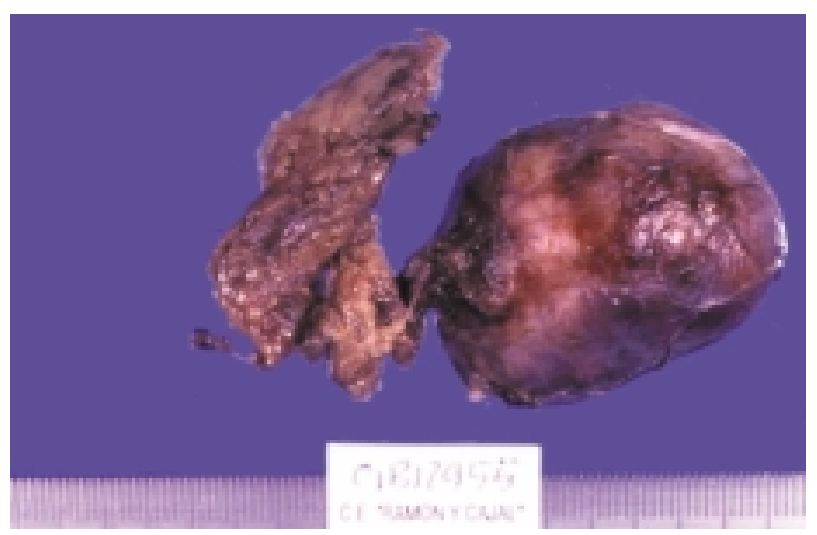

FIGURA 2. Imagen macroscópica de la tumoración: encapsulada, pardo-amarillenta y consistencia elástica.

Micro-tumoración completamente encapsulada (Fig. 3). Se trata de una tumoración mesenquimal de densidad celular variable, constituida por elementos fusocelulares de mediano tamaño sin atipia evidente. En algunas células el citoplasma es ondulado y los núcleos se disponen

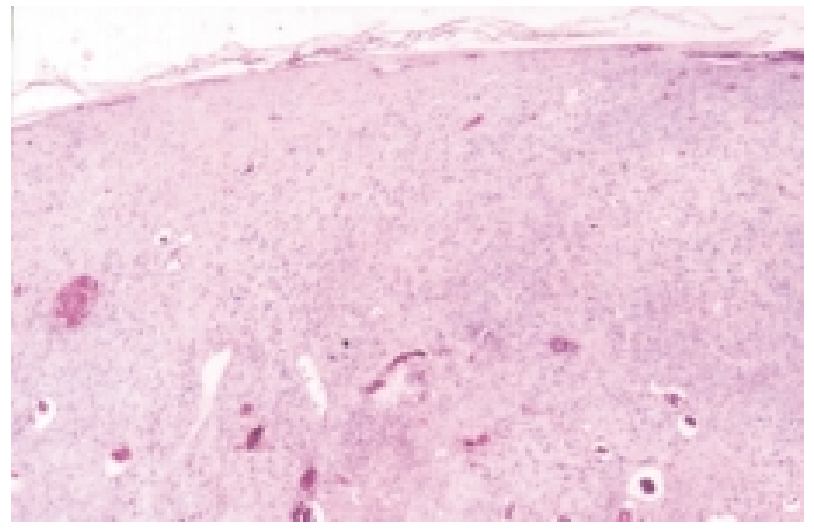

FIGURA 3. Imagen microscópica con tinción hematoxilina-eosilina a 2,5 aumentos: vista panorámica del tumor donde se aprecia proliferación encapsulada de células fusiformes con vasos. 
en empalizada. Destaca la abundancia de vasos con pared hialinizada (Fig. 4). El estudio inmunohistoquímico evidencia la presencia de células tumorales positivas para vimentina y S100 (Fig. 5), que confirma el diagnóstico de tumor benigno de nervio periférico (schwannoma o neurilemoma).

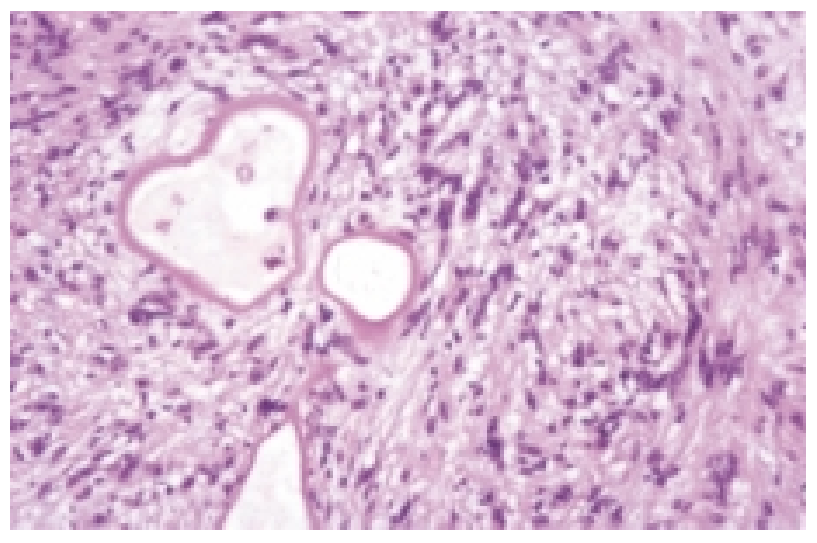

FIGURA 4. Imagen microscópica a 60 aumentos con tinción hematoxilina-eosilina (H-E). Se aprecian vasos con paredes hialinizadas $y$ atipias celulares de caracteristicas degenerativas.

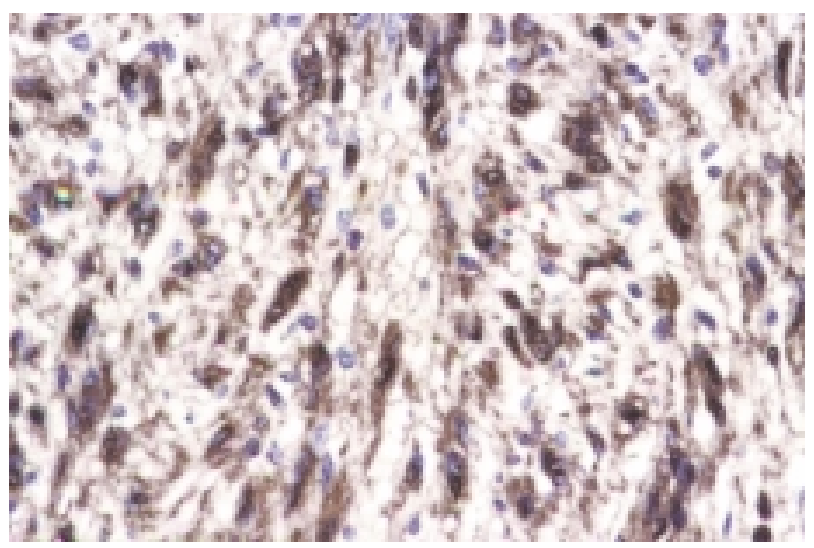

FIGURA 5. Inmunotinción positiva para la proteina S-100.

\section{DISCUSIÓN}

Se presenta este caso por dos motivos, primero la posibilidad de estar ante una segunda neoplasia secundaria al tratamiento de su tumor de Wilms en la infancia y la escasa frecuencia del schwannoma benigno retroperitoneal.

Como hemos comentado en la introducción existe un riesgo mayor que la población general de desarrollar segundos tumores en los niños tratados por tumor de Wilms. Las neoplasias malignas más frecuentes son los sarcomas de partes blandas, tumores óseos (en especial osteosarcomas), hepatocarcinomas, carcinomas tiroideos, leucemias, melanomas y otros. La mayor parte de estos tumores se han desarrollado en zonas correspondientes a los campos utilizados para la aplicación de las radiaciones ${ }^{1}$.

Realizamos una búsqueda bibliográfica en distintas bases de datos (Medline, Silverplate, etc.), y no existen referencias en la literatura en relación con la asociación de schawonnoma tras el tratamiento del tumor de Wilms. Planteamos este caso como una nueva posibilidad de segundo tumor, destacando la importancia de un seguimiento a largo plazo (toda su vida) en pacientes afectos de un tumor de Wilms.

En relación al schawonnoma, es una entidad poco frecuente, no bien registrada en la literatura por la multitud de sinónimos (schwannoma, fibroblastoma, neurinoma y neurilemoma, glioma de nervio periférico, fibroblastoma perineural) empleados, aunque parece que los más adecuados deben ser schwannoma, neurinoma, neurilemoma $^{2-4}$. Fue descrito por primera vez por Verocay en 1910, quien lo denominó neurinoma ${ }^{5}$. La denominación retroperitoneal del schwannoma es comunicada por primera vez en $1954^{6}$.

Se origina a nivel de las células de schwann de nervios periféricos, sobre todo en cuello, cabeza y extremidades ${ }^{2}$.

La edad de presentación oscila entre la $4^{\underline{a}}$ y $7^{\text {a }}$ décadas, afectando por igual a ambos $\operatorname{sexos}^{3,7,8}$.

Clínicamente este tipo de tumores suele ser silente, siendo diagnosticado en la mayoría de las ocasiones de forma casual. Si presenta síntomas suele ser en forma de dolor lumbar sordo; la aparición de síndrome constitucional, hematuria y/o fiebre sugieren la forma maligna ${ }^{2,3}$.

Las técnicas de imagen más empleadas para el diagnóstico son la ecografía y la TAC, presentando como datos más específicos (63\% de los tumores benignos), un área central de aspecto quístico que parece guardar relación con fenómenos de proliferación e hialinización endotelial vascular ${ }^{8,9}$.

La PAAF de estas lesiones no suele aportar certeza diagnóstica por el pleomorfismo celular propio de estos tumores y por su escasa frecuencia, confundiendo al patólogo en muchas ocasio- 
nes; por tanto el estudio histopatológico e inmunohistoquímico nos aportarán el diagnóstico de certeza. Algunos autores justifican su realización porque esta técnica permite distinguir de otros procesos retroperitoneales (abscesos, hematomas, otro tipo de tumores, etc.). En este caso se creyó necesaria su realización ante la posibilidad de recidiva de su tumor de Wilms ${ }^{10,13}$

Suelen ser tumores solitarios, de crecimiento lento que comprimen, en vez de invadir, las estructuras anatómicas normales, crecen en el interior de la vaina nerviosa, lo cual permite una disección relativamente sencilla del tumor, sin necesidad de lesionar el nervio ${ }^{3,14}$.

$\mathrm{Su}$ apariencia histológica es característica, generalmente se reconocen dos patrones: el tipo A de Anthony y el tipo B. Las zonas tipo A, son bastante celulares y están constituidas por células fusiformes que con frecuencia se disponen en empalizada o en estructuras organoides (cuerpos de Verocay). En las zonas tipo B, las células tumorales están separadas por un fluido edematoso, formando en ocasiones espacios quísticos ${ }^{2-4,13}$.

El tratamiento consistirá en la extirpación cuidadosa y completa de la lesión. Aunque el pronóstico de los schwannomas benignos retroperitoneales es muy bueno, precisa de un seguimiento a largo plazo porque se han descrito recidivas en relación a la extirpación incompleta de la lesión. También se han descrito casos de malignización, usualmente asociados al síndrome de von Recklinghausen ${ }^{12,15}$

\section{REFERENCIAS}

1. EVANS A, NORKOOL P.: Late effects of treatment for Wilm's tumor. A report for the national Wilms tumour study group. Cancer 1991; 67: 331-336.

2. ESCLARIN DUNY MA, SANZ VÉLEZ JI, ORTAS CANTAN P, MENGUAL GIL P, VERA ÁLVAREZ J, ABAD TOGER D, CARMEN SALINAS D.: Tumor retroperitoneal infrecuente: schwannoma benigno. Actas Urol Esp 1991; 15: 577-579.
3. CORTES ARANGUEZ I, FERNÁNDEZ ARJONA M, DE CASTRO BARBOSA F, DÍAZ FERNÁNDEZ J, DELGADO PORTELA M.: Schwannoma retroperitoneal benigno: revisión de la literatura y aportación de un nuevo caso. Actas Urol Esp 1999; 23 (7): 621-624.

4. TAKATERA H, TAKIUCHI H, NAMIKI M, TAKAHA M, OHNISHI S, SONODA T.: Retroperitoneal schwannoma. Urology 1986; 28: 529-531.

5. VEROCAY J.: Zur Kenntnis der neurofibrome. Beitr Path Anat Allag Path 1910; 48: 1.

6. DEMING C, NEWMAN H.: Schwannomas. $J$ Urol 1954; 72: 316.

7. REGAN JF, JULER GL, SCHMUTZER KL: Retroperitoneal neurilenoma. Am J Surg 1986; 117: 529-531.

8. FÉLIX EL, WOOD DK, GUPTA TK.: Tumours of the retroperitoneum. Curr Probl Cancer 1981; 6: 1.

9. GHIATAS A, FALESKI EJ.: Benig solitary schwannoma of the retroperitoneum: CT features. South Med $J$ 1989; 82: 801.

10. CLAES H, OYEN R, STESSENS R, VEREECKEN R.: Solitary benig schwannoma in the psoas muscle. $J$ Urol 1987; 137 (1): 757.

11. DONNAL JF, BAKER ME, MAHONY BS, LEIGHT GS.: Benign retroperitoneal schwannoma. Urology 1988; 31: 4.

12. STEERS WD, BYRON HODGE G, JOHNSON DE, CHAITIN BA, CHARNSANGAVEJ CH.: Benig retroperitoneal neurilenoma without von Recklinghausen`s disease: a rare ocurrence. $J$ Urol 1985; 133: 5.

13. NEIFER R, NGUYEN GK.: Aspiration cytology of solitary schwannoma. Acta Cytol 1985; 29: 1.

14. GUZ BV, WOOD DP, MONTIE JE, PONTES JE.: Retroperitoneal neureal sheath tumors: Cleveland clinic experience. J Urol 1989; 142: 1434.

15. NAVALON P, CURRELLS LP, FERRER R, TARIN M, BATELLER J, FERRER RODA J.: Schwannoma retroperitoneal: aportación de un caso. Arch Esp Urol 1989; 42: 4.

Dr. B. Pozo Mengual

C/ Camilo José Cela, 4 - portal 2, 2응

28230 Las Rozas (Madrid)

(Trabajo recibido el 17 junio de 2002) 